\title{
Simultaneous measurement of refractive index and temperature using multimode interference inside a high birefringence fiber loop mirror
}

\author{
Carlos Gouveia $^{\mathrm{a}, \mathrm{b}, *}$, Giancarlo Chesini ${ }^{\mathrm{c}}$, Cristiano M.B. Cordeiro ${ }^{\mathrm{c}}$, J.M. Baptista ${ }^{\mathrm{a}, \mathrm{b}}$, Pedro A.S. Jorge ${ }^{\mathrm{a}, * *}$ \\ a INESC Porto, Rua do Campo Alegre, 687, 4169-007 Porto, Portugal \\ b Centro de Competências de Ciências Exactas e de Engenharia, Universidade da Madeira, Campus da Penteada, $9000-390$ Funchal, Portugal \\ ' Instituto de Física “Gleb Wataghin", Universidade Estadual de Campinas - UNICAMP, Campinas, SP, Brazil
}

\section{A R T I C L E I N F O}

\section{Article history:}

Received 7 August 2012

Received in revised form 2 November 2012

Accepted 23 November 2012

Available online $\mathrm{xxx}$

\section{Keywords:}

Fiber loop mirror

Multimode interference

Refractive index measurement

Temperature measurement

Refractive index and temperature

discrimination

Multiparameter

\begin{abstract}
A B S T R A C T
A fiber optic sensor for simultaneous measurement of refractive index and temperature is presented. The sensing probe is realized by introducing a multimode interference device inside a high birefringence fiber loop mirror resulting in a configuration capable of refractive index and temperature discrimination. The multimode interference peak is sensitive to the surrounding refractive index ( $90 \mathrm{~nm} / \mathrm{RIU})$ and slightly responsive to the temperature $\left(0.01 \mathrm{~nm} /{ }^{\circ} \mathrm{C}\right)$. On the other hand, the birefringent fiber loop mirror is highly sensitive to temperature $\left(2.36 \mathrm{~nm} /{ }^{\circ} \mathrm{C}\right)$ and it has almost no response to refractive index. Using a power ratiometric peak detection scheme, a temperature independent refractive index measurement can be achieved with a resolution of $\pm 2.25 \times 10^{-5}$ RIU.
\end{abstract}

(C) 2012 Elsevier B.V. All rights reserved.

\section{Introduction}

The increasing need for integration and miniaturization has driven researchers toward developing smaller and more efficient optical sensors. Among several physical parameters under observation, one of particular interest is the refractive index (RI). Its measurement is important for chemical and biological sensing in medical, industrial and environmental applications. In such cases label free optical sensing, based on the measurement of RI, represents a viable alternative to standard techniques, usually based on an indicator dye. Refractometric based approaches do not interfere with the analyte properties and require, instead, the design of sensitive layers that experience a refractive index changes in its presence [1].

Fiber optic sensors, in particular, present an interesting solution in this context due to their feasible integration to other fiber optics components, high sensitivity, small size, and capability for in-situ, real-time, remote, and distributed sensing. Refractive index sensing using optical fibers has already been proposed and demonstrated in several ways. A simple fiber optic technique based on Fresnel

\footnotetext{
* Corresponding author at: INESC Porto, Rua do Campo Alegre, 687, 4169-007 Porto, Portugal. Tel.: +351 220402 301; fax: +351 220402437 .

** Corresponding author.

E-mail address: cgouveia@inescporto.pt (C. Gouveia).
}

reflection from the fiber tip was explored for RI measurements in liquids through the ratio of the reflected signals from the fiberair and from the fiber-liquid interfaces [2,3]. Fiber tapers have an enhanced evanescent interaction and have been widely explored for RI measurements by monitoring the transmitted optical power [4]. In spite of high sensitivity, however, these structures are very fragile.

Optical fiber gratings, including fiber Bragg gratings (FBGs) and long-period fiber gratings (LPGs), have also been explored for RI measurements. They consist in a periodic modulation of the refractive index of the core of the fiber, where the LPG's period is much longer (hundreds of microns) than the FBG's period (typically a half-wavelength). This structural difference results in devices with fundamentally different properties. FBG work mainly with radiation confined to the fiber core, this way strategies have to be devised in order for the radiation to interact with the external medium. Several refractometers based in Bragg gratings have been studied, typically, FBG based refractometers rely on the evanescent field of the core modes under fiber etching conditions $[5,6]$. Nevertheless, these configurations introduce fragility in the fiber sensor. Alternative techniques using tilted FBGs [7] and FBG cladding modes [8] were also presented. These sensing schemes are also based on evanescent interaction. However, working with FBG cladding modes that are spectrally not so well resolved, introduces difficulties in the accurate interrogation of the sensors. 


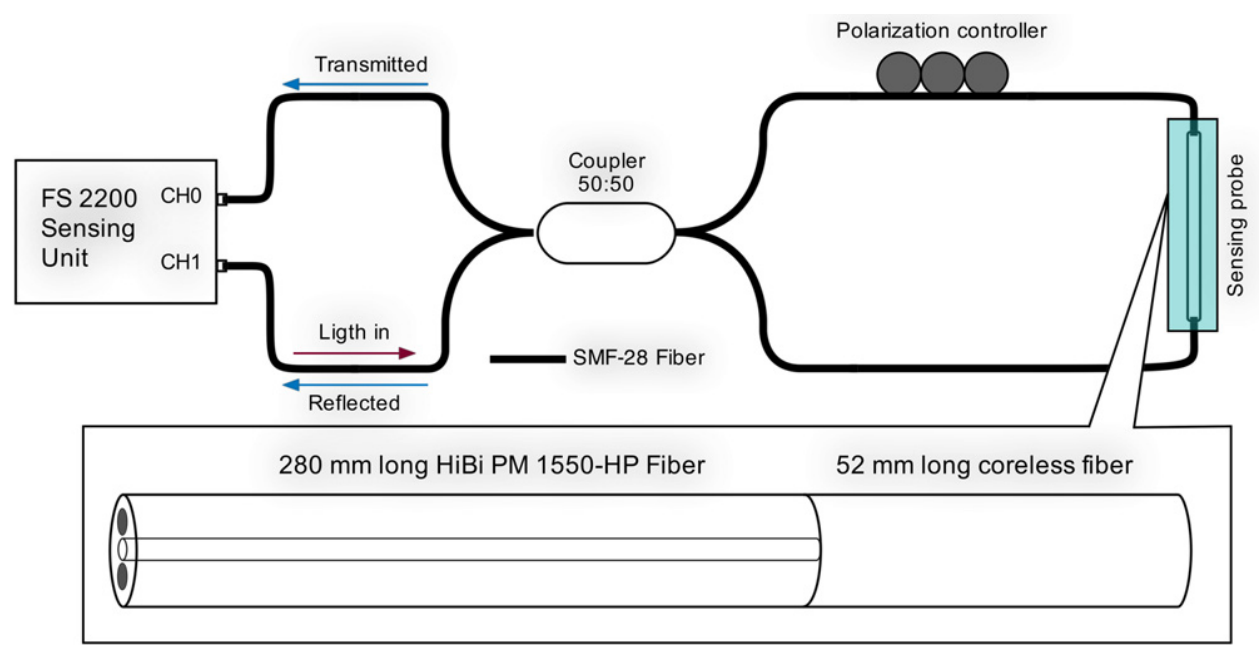

Fig. 1. Experimental setup and sensing probe schema.

Long period gratings (LPG), on the other hand, provide evanescent interaction by exciting cladding modes, and are therefore intrinsically sensitive to external refractive index changes. They maintain fiber integrity and are also widely used for RI measurement, by using a bare LPG [9] or using an LPG based interferometric scheme [10]. However, because they are also highly sensitive to temperature they need an extra mechanism to compensate temperature changes.

Another type of fiber refractometers based on metal nano layers has been developed, where the thin film properties create surface plasmon resonance (SPR) or lossy modes resonances (LMRs), which act as the sensing mechanism. In both cases the metallic layer induces losses in the propagation of light in the fiber. SPR occurs when the real part of the thin film permittivity is negative and higher in magnitude than both its own imaginary part and the permittivity of the material surrounding the thin film [11]. LMRs occurs when the real part of the thin film permittivity is positive and higher in magnitude than both its own imaginary part and the material surrounding the thin film [12]. In any case, a coating has to be deployed over the fiber structure to ensure a good evanescent interaction like the one that occurs with tapers or unclad fibers. These kinds of sensors are very sensitive to external RI and are very appealing for biosensing applications. However, they typically work in transmission mode and present very broad spectral resonances making multiplexing a very hard task.

Multimode interference (MMI) based refractometers are also interesting solutions that rely on the concept of re-imaging effects of MMI patterns present in multimode waveguides. In these devices, the transmitted spectral power distribution is highly sensitive to the optical path length of the multimode fiber and its surrounding RI. Different MMI based refractometers have been proposed, showing robustness and good sensitivity [13-16].

High-Birefringence fiber loop mirrors (HiBi FLM), on the other hand, are interesting devices that usually consist on a section of $\mathrm{HiBi}$ fiber that is spliced between the output ports of a directional optical coupler to obtain an optical path difference between the fast and the slow axis. Independence of input polarization and insensitivity to perturbations in the lead in fibers are some of its most attractive features. FLM have been widely used in the optical fiber sensor field mostly for the measurement of physical parameters [17] and due its high temperature sensitivity, this structure was also presented for temperature discrimination [18]. Despite its extensive use in the measurement of physical parameters, however, only a few works were presented to measure RI with FLM. Usually this is achieved by introducing an RI sensitive structure inside the loop such as
D-type fiber [19] an LPG written in a polarization maintaining fiber [20], a non-adiabatic fiber taper [21] and more recently using two sections of $\mathrm{HiBi}$ fiber with different lengths into a FLM, were used for differential RI measurement [22], being one of the sections partially etched.

In the present work a sensing structure capable of simultaneous measurement of refractive index and temperature is demonstrated by combining the strengths of two well-known fiber sensing devices. The sensing probe consists in a coreless multimode fiber segment inserted into a HiBi FLM. The multimode interference device introduces advantages such as low cost, ease fabrication and good RI sensitivity while keeping the fiber structure integrity, unlike other types of FLM RI [19,21]. When the MMI is inserted into $\mathrm{HiBi}$ FLM, on the other the ability to measure temperature with high sensitivity is added. Thus, simultaneous measurement of refractive index and temperature is achieved with high resolution, paving the way for temperature independent label free bio-chemical sensors.

\section{Experiment}

Fig. 1 shows the experimental setup for simultaneous measurement of refractive index and temperature. The sensing head consists in a fiber loop mirror, containing a section of HiBi PANDA fiber and an MMI structure. The sensing element was characterized using a FS 2200 Braggmeter (Fibersensing, SA) working in the $1500-1600 \mathrm{~nm}$ range, with $1 \mathrm{pm}$ resolution, and modified to measure signals both in reflection and transmission modes in separate channels.

The HiBi FLM is formed by a 50:50 $(2 \times 2)$ optical coupler with low insertion loss, an optical polarization controller (PC) and the HiBi single mode fiber section. The PANDA optical fiber (PM1550$\mathrm{HP}$ ), from Thorlabs, $\mathrm{GmbH}$, is a polarization maintaining fiber for a wavelength of $1550 \mathrm{~nm}$ with a beat length of $<5.0 \mathrm{~mm}$ and an attenuation of $<1 \mathrm{~dB} / \mathrm{km}$. The HiBi FLM works as periodic bandpass filter for the input signal. The input optical signal is splitted into two counter-propagating beams, which undergo the loop crossing the PC before or after propagating the PANDA fiber. This asymmetry swaps the polarization components of both clockwise and counterclockwise beams in such a way that, at the outputs, the two waves have an accumulated phase difference that is proportional to the difference in optical paths of the fast and slow axis of the HiBi section, resulting in constructive or destructive interference, depending on the relative phase at recombination after crossing the coupler ports. The result is a fringe pattern whose periodicity is proportional to the HiBi fiber length and its birefringence. 
Due to the dependence of the Birefringence of this Panda fiber with the temperature, the fringe pattern will be highly sensitive to temperature. The fringe period is given by $\Delta \lambda=\lambda^{2} / \beta L$, where $\lambda$ is the average wavelength and $\beta$ and $L$, the birefringence and the length of the HiBi fiber, respectively. The length used for the HiBi fiber was approximately $280 \mathrm{~mm}$, resulting in a fringe pattern with a $30 \mathrm{~nm}$ periodicity.

The sensing probe for refractive index and temperature discrimination is based on a combination of the HiBi FLM and an MMI device. The MMI was formed by splicing a section of pure silica coreless multimode fiber (CMF) to the PANDA fiber. The CMF was fabricated by drawing a silica rod down to the appropriate dimensions in a standard optical draw tower. Singlemode fibers, SMF-28, with core and cladding diameters of 8.2 and $125 \mu \mathrm{m}$, respectively, were used for both light input and output of the PANDA-CMF section. In this situation, the CMF section behaves like a standard MMI device. At the input of the CMF several high order modes are excited generating a periodic interference pattern along the coreless section. Depending on the wavelength and geometrical length the light into de CMF can interfere constructively or destructively resulting, at the end, in a device with different spectral characteristics. Therefore the length of the CMF determines the spectral features of the MMI device. Depending where the interference pattern is 'intersected', constructive or destructive interference results, at different wavelengths yielding the transmission of resonant peaks or resonant losses respectively. Independently of its shape, each of these spectral features will shift according to the surrounding RI with a similar sensitivity [14]. In the present work, a CMF with a diameter of $125 \mu \mathrm{m}$ and a length of $52 \mathrm{~mm}$ was used to form the MMI structure. This length was chosen, based on previous experience [15], to obtain a resonance dip centered at a wavelength of $1525 \mathrm{~nm}$ with a maximum loss of $25 \mathrm{~dB}$ and a full width half maximum (FWHM) of approximately $10 \mathrm{~nm}$.

Prior to insertion of the sensing device into the FLM, its transmission spectra was observed by injecting light from either side, to make sure no asymmetry was introduced by the splicing process. The device spectral output was shown to be independent of the direction of observation demonstrating a good symmetry. Further preliminary tests were made to evaluate the degree of cross interference between the sensing properties of the two devices combined in this work. The MMI device was first inserted into a FLM, with no Hi-Bi fiber. In this case no interference effects were observed, showing that the device had no birefringent properties. A resonant loss was observed with identical characteristics to what was observed outside the loop. On the other hand, it was verified that the Hi-Bi interference pattern of the combined sensing device was not sensitive to refractive index, within the measurement error. This way, for the present experiment it was assumed that the working principles of each of the devices were operating in an independent way.

Fig. 2 shows the transmitted (A) and reflected (B) spectra of the sensing element, when inserted into the FLM. It is possible to appreciate the periodic bandpass filter created by the loop superimposed with the MMI resonances. The MMI peak is sensitive to the RI solution and slightly responsive to temperature. Conversely the wavelength fringes formed by the loop mirror are highly sensitive to temperature and practically do not respond to RI. The MMI interference pattern, being non birefringent is common to both polarization modes and it is therefore equally observable both in the transmitted and reflected signals. Its spectral characteristics are identical to what was observed prior to insertion into the loop, confirming no cross interference between the two sensitive elements. The HiBi segment, on the other hand, generates a fringe pattern whose visibility depends on the amount of radiation propagating in each birefringence axis. This way, using the PC it was possible to control the amount of light traveling in each polarization mode,
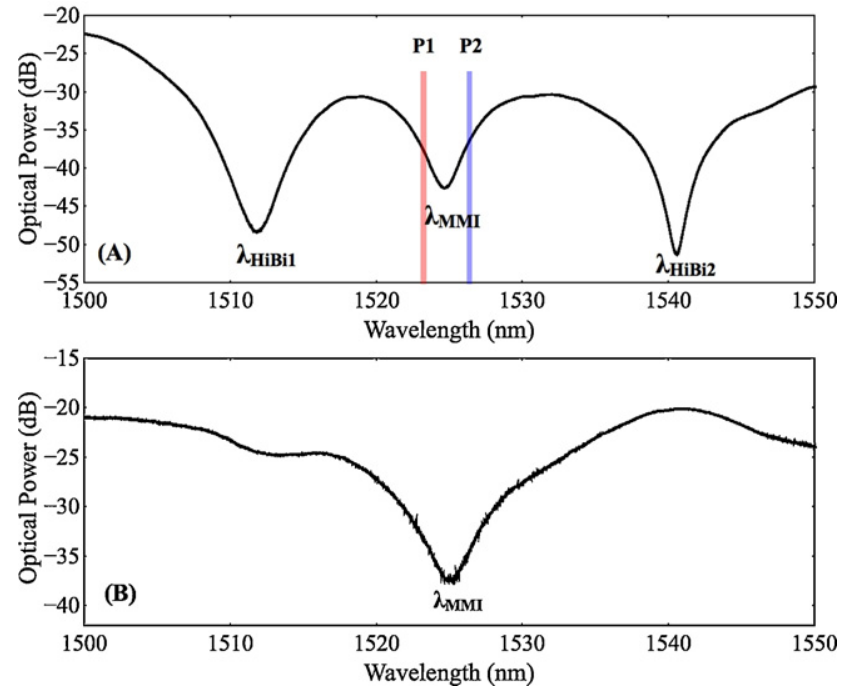

Fig. 2. Transmitted (A) and reflected (B) spectra of the HiBi FLM-MMI device.

and therefore control the relative visibility of the reflected and transmitted signals. In the transmitted signal (A), one can see the superposition of the HiBi interference pattern and the MMI interference pattern. On the reflected signal (B), on the other hand, since the visibility of the HiBi pattern was reduced to zero, by further adjusting the PC, only the MMI interference pattern is visible.

In order to enhance the tracking resolution of the RI induced wavelength shifts we implemented a ratiometric detection scheme. LabView control of the FS 2200 enabled real-time direct access and processing of the data sets, corresponding to both reflection and transmission signals. Two narrow spectral intervals were chosen at the each side of the resonant dip (shown in Fig. 2(A)). The integrated optical power of each wavelength slit resulted in signals $P_{1}$ and $P_{2}$. The subsequent computation of $R=\left(P_{1}-P_{2}\right) /\left(P_{1}+P_{2}\right)$ yields a signal that is proportional to the wavelength shift and is independent of any optical power fluctuation, providing higher accuracy than standard peak tracking schemes [23]. It should be taken into consideration that the wavelength shift range of this algorithm is limited by the spectral position of the selected intervals. In fact this operation is suitable and intended to measure very small variation of a resonant peak with high accuracy. In such measurement ranges, changes in the resonance FWHM, which could otherwise cause measurement errors, are practically negligible. For the present experiment only the transmitted spectrum was considered, the $R$ parameter was used to monitor with high resolution the MMI peak $\left(\lambda_{\mathrm{MMI}}\right)$. The wavelength of the second peak of the $\mathrm{HiBi}$ $\left(\lambda_{\mathrm{HiBi} 2}\right)$, presenting much larger wavelength shifts, was recorded using a standard peak tracking algorithm.

\section{Results and discussion}

Refractive index and temperature sensitivities were characterized separately. To avoid strain/curvature cross sensitivity, in both experiments, the sensing head was properly fixed and in the first case, placed inside a test chamber. The RI response was characterized by exposing the sensor head to different solutions of distilled water with different percentages of salt at constant temperature $\left(25^{\circ} \mathrm{C}\right)$. Each liquid sample had its RI previously characterized with an Abbe refractometer using the sodium $\mathrm{D}$ line $(589 \mathrm{~nm})$. We studied solutions with a RI in a range from 1.3360 (pure distilled water) to $1.3440 \mathrm{RIU}$. The necessary adjustments, considering the sensing head operation at $1550 \mathrm{~nm}$, can be made using the Cauchy equation with the respective coefficients [5]. 


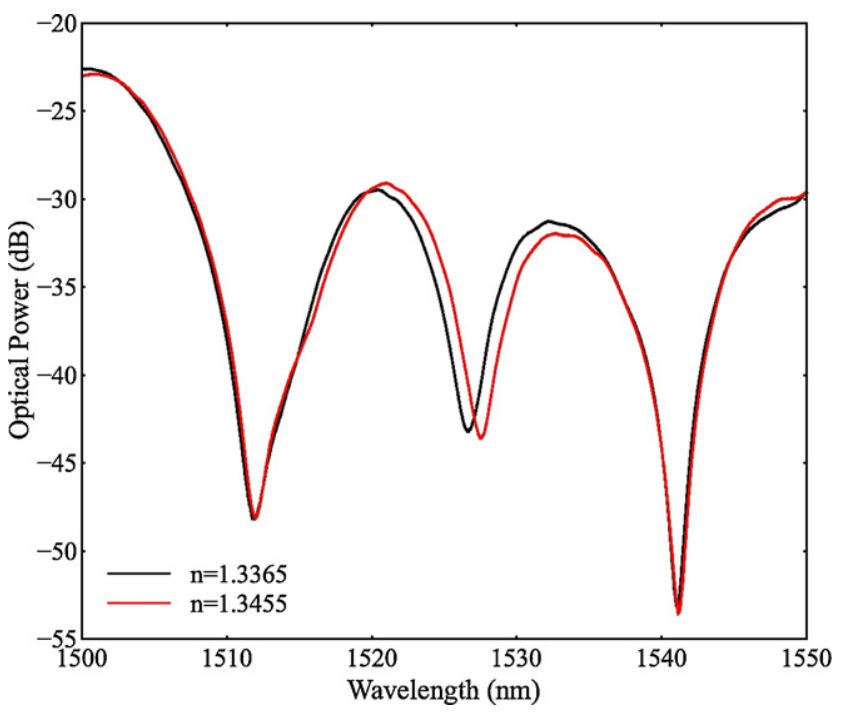

Fig. 3. Transmitted spectra of the sensing probe for two different surrounding refractive indices.

The transmitted spectra for two different solutions of salty water are shown in Fig. 3. As expected the MMI resonance shifts toward longer wavelengths as we increase the refractive index, i.e. the salt percentage. Meanwhile the HiBi FLM peaks remain practically unaltered (only a small fluctuation with standard deviation of $15 \mathrm{pm}$ was appreciable in the studied range). Using a standard LabView peak detection algorithm it was possible to track the wavelength peak for different values of refractive index and estimate a sensitivity of $90 \mathrm{~nm} / \mathrm{RIU}$ from the slope of the collected data. The real time resolution of the system was also estimated by applying a very small step change in the sample refractive index. From the statistics of the measured values the standard deviation was calculated for each step. It was then considered a minimum detectable signal of two times the largest calculated standard deviation $(\sigma)$ in the measurement interval. From this procedure a refractive index resolution of $\pm 2.5 \times 10^{-4}$ RIU could be estimated.

As discussed earlier, to enhance the tracking resolution, a ratiometric power algorithm was implemented. Fig. 4 shows the behavior of the $R$ parameter as a function of the solution RI in the

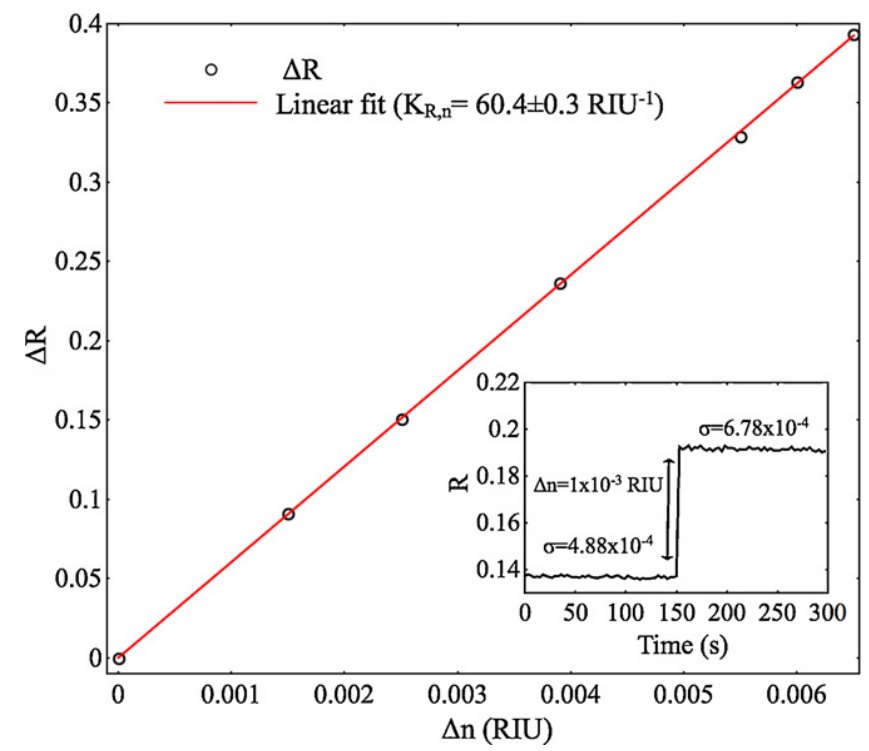

Fig. 4. $R$ parameter as a function of the variation of the refractive index. Inset, variation of $R$, induced by a step change of the surrounding RI.

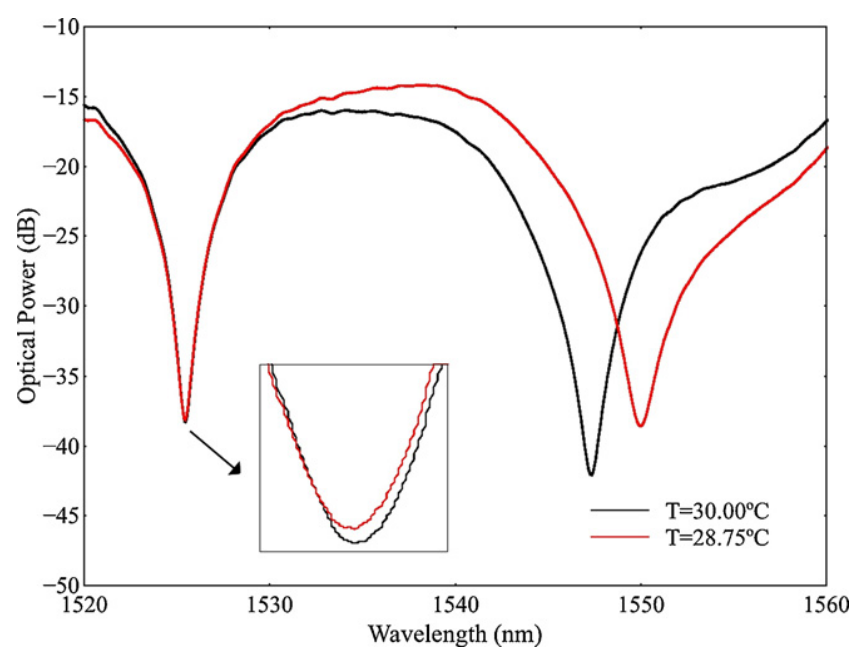

Fig. 5. Transmitted spectra of the sensing probe for two different temperatures.

studied range. A sensitivity of $60.4 \pm 0.3 /$ RIU can be estimated from the slope of the acquired data. The inset in Fig. 4 illustrates the dynamic behavior of $R$, induced by a step change of $1 \times 10^{-3}$ in the RI. From the statistics of the real time retrieved data, the standard deviation was calculated for each step. It was then considered a minimum detectable signal of two times the largest calculated standard deviation $(\sigma)$ in the measurement interval. From this procedure a refractive index resolution of $\pm 2.25 \times 10^{-5}$ RIU was estimated. In addition, from the statistics of the linear regression, resulting for a much larger number of measurements, the estimated $2 \sigma$ resolution was of $\pm 2.42 \times 10^{-5} \mathrm{RIU}$, demonstrating the measurement reproducibility. $2 \sigma$ was considered in order to facilitate comparison with other refractometer in literature such as [23] and [24]. In a practical application more stringent statistics can be applied. In such case, for instance a $3 \sigma$ standard would yield a resolution of $\pm 3.62 \times 10^{-5}$ RIU.

For temperature characterization, the sensing head was placed over a hot plate. The temperature was measured using a thermocouple. The temperature was changed between $28.75^{\circ} \mathrm{C}$ and $30^{\circ} \mathrm{C}$. Fig. 5 shows transmitted spectrum for a variation of temperature of $1.25^{\circ} \mathrm{C}$. As expected, with the increment of the temperature the HiBi peaks shift to shorter wavelengths. Simultaneously, it is noticeable a very small change in the MMI resonance toward longer wavelengths (inset of Fig. 5)

Fig. 6 shows the calibration curves resulting from the thermal studies. For the second peak of the HiBi FLM a sensitivity of $-2.36 \pm 0.06 \mathrm{~nm} /{ }^{\circ} \mathrm{C}$ was measured. The $R$ parameter of the MMI peak, on the other hand, varies $0.078 \pm 0.001 /{ }^{\circ} \mathrm{C}$. This means that even for a temperature fluctuation of $0.1^{\circ} \mathrm{C}$ an error of at least $10^{-4}$ RIU will be introduced. This way, for biosensing application, where high refractive index resolution is mandatory, and considering the resolution of this MMI sensor, simultaneous measurement of temperature must be performed in order to determine the refractive index without ambiguity.

In Table 1 , it is summarized the sensitivity coefficients of the $\mathrm{HiBi}$ FLM-MMI sensor system for refractive index $\left(K_{n}\right)$ and temperature $\left(K_{T}\right)$ taken from the slopes of the lines represented in Figs. 4 and 6.

Table 1

Sensitivity coefficients of the sensing probe for refractive index and temperature.

\begin{tabular}{lcl}
\hline & $K_{n}$ & $K_{T}$ \\
\hline$\Delta R$ & $60.4 \pm 0.3 / \mathrm{RIU}$ & $0.078 \pm 0.001 /{ }^{\circ} \mathrm{C}$ \\
$\Delta \lambda$ & - & $-2.36 \pm 0.06 \mathrm{~nm} /{ }^{\circ} \mathrm{C}$ \\
\hline
\end{tabular}



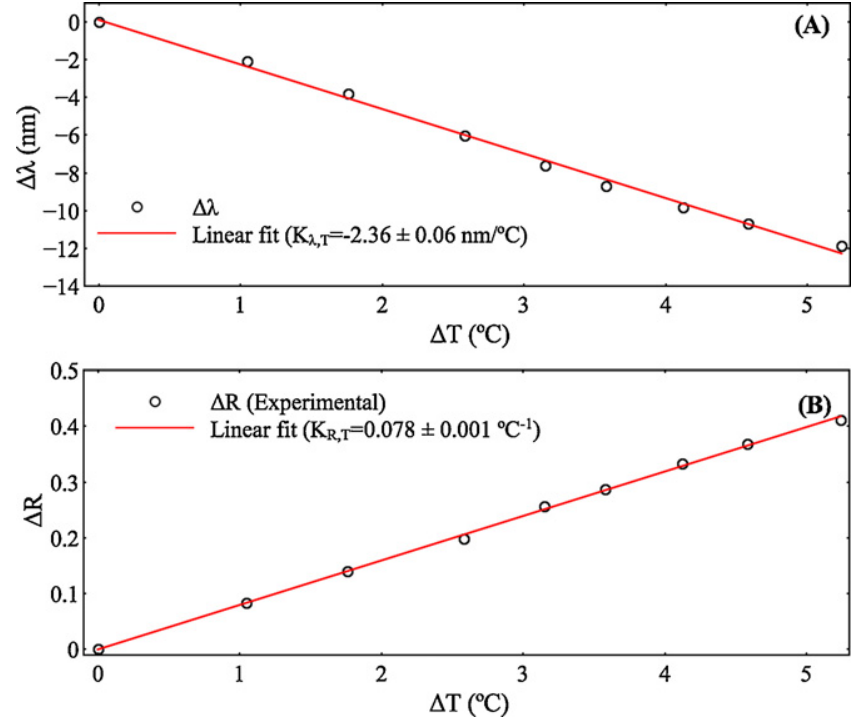

Fig. 6. Sensing probe behavior as a function of temperature variations. (A) Resulting from the second peak of the HiBi fringes and (B) resulting from the R parameter of the MMI peak.

The dual response of the MMI $R$ parameter and the shift, $\Delta \lambda$, of the second peak of the FLM, allows writing a conditioned system of two equations for $\Delta n$ and $\Delta T$, given in matrix form as;

$$
\left[\frac{\Delta T}{\Delta n}\right]=-\frac{1}{K_{\lambda, T} K_{R, n}}\left[\begin{array}{cc}
0 & K_{R, n} \\
K_{\lambda, T} & -K_{R, T}
\end{array}\right]\left[\frac{\Delta R}{\Delta \lambda}\right]
$$

where $K_{n, R}, K_{T, R}$ and $K_{T, \lambda}$, the matrix elements, are the sensitivity coefficients of the sensing probe represented in Table 1.

$$
\left[\frac{\Delta T}{\Delta n}\right]=-\frac{1}{142.544}\left[\begin{array}{cc}
0 & 60.4 \\
-2.36 & -0.078
\end{array}\right]\left[\frac{\Delta R}{\Delta \lambda}\right]
$$

With these tools temperature and refractive index can be determined unequivocally by processing the signals retrieved from the HiBi FLM and MMI sensing head. The system performance was evaluated when the sensing probe was simultaneously subjected to RI and temperature changes. In order to change both measurands a solution of ethylene glycol was used. When added to distilled water, ethylene glycol produces an exothermic reaction and consequently the increment of the solution temperature. Simultaneously, a variation of RI comes from the different concentrations of ethylene glycol. The measured raw signals and those processed through the resulting equations from the matrix are shown in Fig. 7. Traces (A) and (B) correspond, respectively, to the raw signals given by the $\mathrm{HiBi}$ peak and the $R$ parameter of the MMI peak. Traces (C) and (D), on the other hand, represent, respectively, the temperature and refractive index information retrieved through the matrix operations. Trace (A) shows a wavelength shift that mimics the gradual increment in temperature as the glycol is added. Since the HiBi signal just depends on temperature, the temperature retrieved by the matrix operation (trace (C)) exactly matches the wavelength behavior. It is clearly visible a sharp increment in temperature when the solution is spiked, followed by a slower cooling to an equilibrium temperature, as the solution is homogenized. The final temperature, nevertheless, is always higher than the original value, resulting in step increments of the average temperature.

Trace (B), given by the $R$ parameter of the MMI peak, depends both on temperature and refractive index. This way, the observed behavior results from the mixed response to these two parameters. However, applying the matrix operations the refractive index behavior, alone, can be retrieved and is represented in trace (D). It

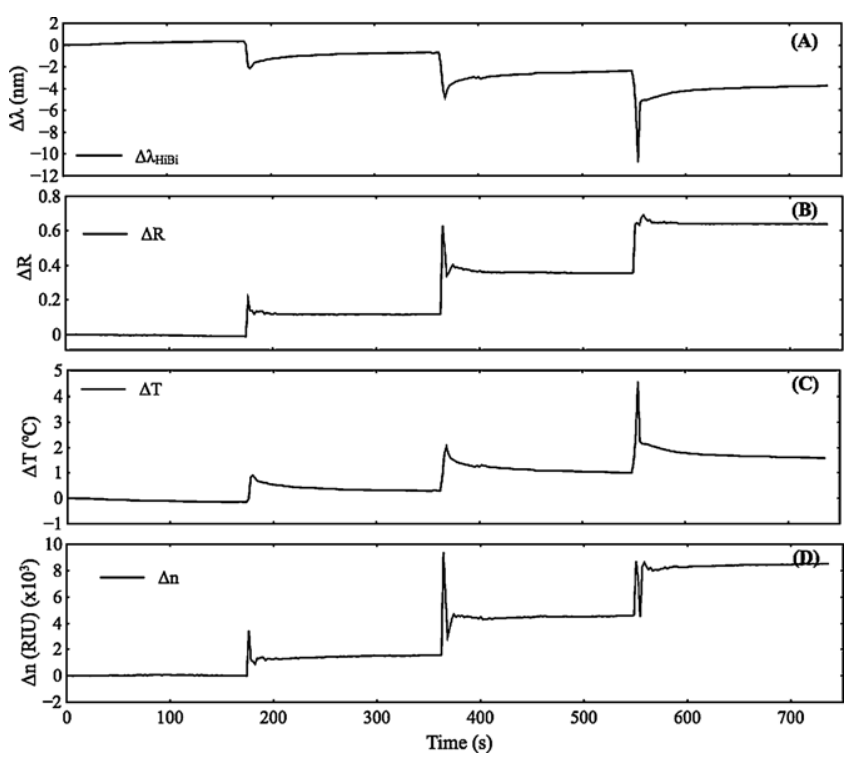

Fig. 7. Sensor output for a simultaneous variation of refractive index and temperature. (A) Wavelength shift of the HiBi peaks, proportional only to temperature, (B) evolution of the $R$ parameter, proportional both to temperature and refractive index, (C) temperature measurement retrieved using data from (A) and (B) and the matrix operations and (D) refractive index measurement retrieved using data from $(A)$ and (B) and the matrix operations.

can be seen that in right after the solution spiking sharp peaks are also observed in the refractive index trace. At this stage of the process, the solution is not homogeneous, so there is the chance the temperature and refractive index sensors are not submitted exactly to the same conditions. In addition, refractive index of the solution is also dependent on its temperature via its thermo optic coefficient. This way, while refractive index spikes may indeed occur, prior to the solution homogenization, the temperature compensation performed by the matrix operation may not be totally effective. Nevertheless, at the equilibrium stage, where the solution has a more homogenous temperature and refractive index distribution, the compensation is indeed effective.

This can be verified in Fig. 8 that shows the recovered temperature and refractive index at different times, considering the average values or the "steady state" value of each incremental step. Independent temperature and RI measurements of the solution using a thermocouple and Abbe refractometer are also shown. The

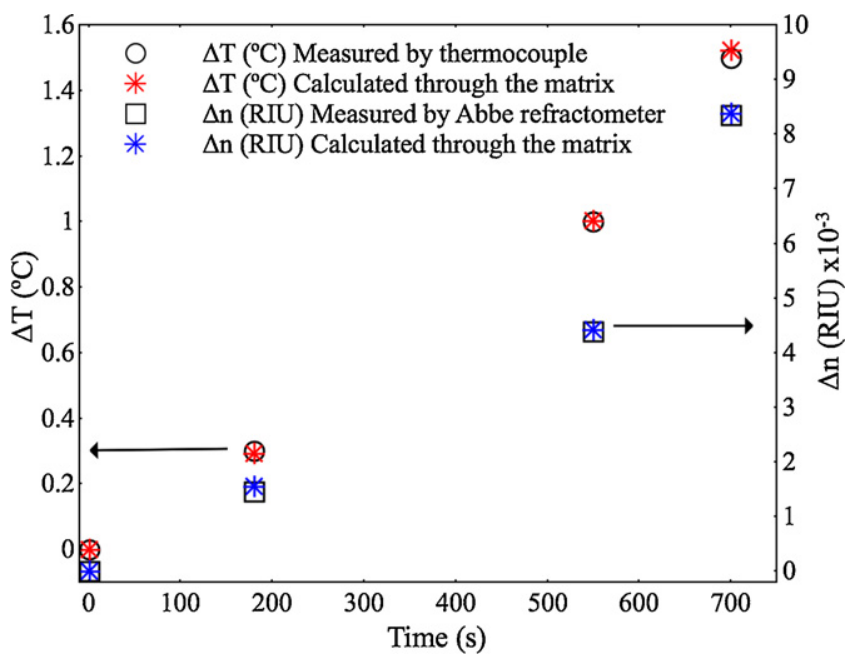

Fig. 8. Measured and recovered data by using the matrix method. 
maximum errors for temperature and RI measurements were found to be $\pm 0.02{ }^{\circ} \mathrm{C}$ and $\pm 2.25 \times 10^{-5}$ RIU respectively.

This way, the proposed sensing system demonstrates to be suitable for high sensitivity refractometric measurement without temperature ambiguity. The RI sensitivity can be further increased (doubled) by using a smaller diameter coreless multimode fiber as it was recently demonstrated in a previous work $[15,16]$. Also the sensing probe length can be reduced using a smaller section of $\mathrm{HiBi}$ fiber, in order to have just one peak in the $1500-1600 \mathrm{~nm}$ window or by using a different polarization maintaining fiber with higher birefringence. The smaller sensing head will be less prone to be affected by non-homogeneous distributions in the probed solutions.

\section{Conclusions}

We have demonstrated a fiber refractometer based on a MMI coreless multimode fiber section inserted into a HiBi FLM. The MMI peak is sensitive to the RI solution ( $90 \mathrm{~nm} / \mathrm{RIU}$ ) and slightly responsive to temperature $\left(0.01 \mathrm{~nm} /{ }^{\circ} \mathrm{C}\right)$. Conversely the external wavelength fringes formed by the loop mirror are highly sensitive to temperature $\left(-2.36 \mathrm{~nm} /{ }^{\circ} \mathrm{C}\right)$ and practically do not respond to RI. The sensor system is capable to detect variation of refractive index in the order of $\pm 2.25 \times 10^{-5}$ RIU.

The system performance was evaluated when the refractometer was simultaneously subjected to refractive index and temperature changes over ranges of 0.08 RIU and $1.5^{\circ} \mathrm{C}$, respectively. The maximum error was found to be $\pm 2.25 \times 10^{-5}$ RIU and $\pm 0.02^{\circ} \mathrm{C}$, correspondingly. The system presented, based in a refractive index and temperature fiber optic sensor is a platform for both chemical and biological sensing applications. For instance, direct measurements of refractive index were shown to provide valuable information on drug/DNA interaction, or cell growth [25,26]. On the other hand, a variety of silica surface functionalization is described in the literature that will render a refractometer a powerful biosensor [1]. The present configuration eliminates the need for very stringent temperature control in such applications allowing for more reliable refractive index based chemical and biochemical monitoring.

\section{Acknowledgments}

This work was partially supported by a bi-national cooperation project, CAPES-FCT Brazil/Portugal and by project AQUAMONITOR n ${ }^{\circ}$ FCOMP-01-0124-FEDER-013911 (Ref. PTDC/AAC$\mathrm{AMB} / 112424 / 2009)$. Carlos Gouveia would like to acknowledge the financial support of FCT (SFRH/BD/63758/2009).

\section{References}

[1] X.D. Fan, I.M. White, S.I. Shopova, H.Y. Zhu, J.D. Suter, Y.Z. Sun, Sensitive optical biosensors for unlabeled targets: a review, Analytica Chimica Acta 620 (2008) $8-26$.

[2] C.B. Kim, C.B. Su, Measurement of the refractive index of liquids at 1.3 and 1.5 micron using a fibre optic Fresnel ratio meter, Measurement Science and Technology 15 (2004) 1683-1686.

[3] A. Suhadolnik, A. Babnik, J. Mozina, Optical-fiber reflection refractometer, Sensors and Actuators B: Chemical 29 (1995) 428-432.

[4] A. Kumar, T.V.B. Subrahmanyam, A.D. Sharma, K. Thyagarajan, B.P. Pal, I.C. Goyal, Novel refractometer using a tapered optical fiber, Electronics Letters 20 (1984) 534-535.

[5] D.A. Pereira, O. Frazao, J.L. Santos, Fiber Bragg grating sensing system for simultaneous measurement of salinity and temperature, Optical Engineering 43 (2004) 299-304.

[6] A. Asseh, S. Sandgren, H. Ahlfeldt, B. Sahlgren, R. Stubbe, G. Edwall, Fiber optical Bragg grating refractometer, Fiber and Integrated Optics 17 (1998) 51-62.

[7] G. Laffont, P. Ferdinand, Tilted short-period fibre-Bragg-grating-induced coupling to cladding modes for accurate refractometry, Measurement Science and Technology 12 (2001) 765-770.

[8] M. Han, F.W. Guo, Y.F. Lu, Optical fiber refractometer based on cladding-mode Bragg grating, Optics Letters 35 (2010) 399-401.
[9] H.J. Patrick, A.D. Kersey, F. Bucholtz, Analysis of the response of long period fiber gratings to external index of refraction, Journal of Lightwave Technology 16 (1998) 1606-1612.

[10] T. Allsop, R. Reeves, D.J. Webb, I. Bennion, R. Neal, A high sensitivity refractometer based upon a long period grating Mach-Zehnder interferometer, Review of Scientific Instruments 73 (2002) 1702-1705.

[11] N. Díaz-Herrera, A. Gonzalez-Cano, D. Viegas, J.L. Santos, M.C. Navarrete, Refractive index sensing of aqueous media based on plasmonic resonance in tapered optical fibres operating in the $1.5 \mu \mathrm{m}$ region, Sensors and Actuators B: Chemical 146 (2010) 195-198.

[12] C.R. Zamarreno, P. Sanchez, M. Hernaez, I. Del Villar, C. FernandezValdivielso, I.R. Matias, F.J. Arregui, Dual-peak resonance-based optical fiber refractometers, IEEE Photonics Technology Letters 22 (2010) $1778-1780$.

[13] H. Fukano, Y. Matsumoto, S. Taue, High-sensitivity optical fiber refractive index sensor using multimode interference structure, IEICE Electronics Express 9 (2012) 302-306.

[14] Y. Jung, S. Kim, D. Lee, K. Oh, Compact three segmented multimode fibre moda interferometer for high sensitivity refractive-index measurement, Measurement Science and Technology 17 (2006) 1129-1133.

[15] S. Silva, E.G.P. Pachon, M.A.R. Franco, J.G. Hayashi, F.X. Malcata, O. Frazao, P. Jorge, C.M.B. Cordeiro, Ultrahigh-sensitivity temperature fiber sensor based on multimode interference, Applied Optics 51 (2012) 2542.

[16] C. Biazoli, S. Silva, M.A.R. Franco, O. Frazao, C.M.B. Cordeiro, Multimode interference tapered fiber refractive index sensors, Applied Optics 51 (24) (2012) 5941-5945, http://dx.doi.org/http://dx.doi.org/10.1364/AO.51.005941.

[17] Y. Zhao, H.K. Wu, Q. Wang, Advances of high-birefringence fiber loop mirror sensors, Journal of Optoelectronics and Advanced Materials 13 (2011) 457-465.

[18] O. Frazao, L.M. Marques, S. Santos, J.M. Baptista, J.L. Santos, Simultaneous measurement for strain and temperature based on a long-period grating combined with a high-birefringence fiber loop mirror, IEEE Photonics Technology Letters 18 (2006) 2407-2409.

[19] O. Frazao, B.V. Marques, P. Jorge, J.M. Baptista, J.L. Santos, High birefringence Dtype fibre loop mirror used as refractometer, Sensors and Actuators B: Chemical 135 (2008) 108-111.

[20] J. Kang, X.Y. Dong, C.L. Zhao, Z.X.Zhang, S.Z.Jin, Measurement of refractive index with a PM-LPG based Sagnac loop sensor, Spectroscopy and Spectral Analysis 31 (2011) 902-905.

[21] M.I. Zibaii, O. Frazao, H. Latifi, P.A.S. Jorge, Controlling the sensitivity of refractive index measurement using a tapered fiber loop mirror, IEEE Photonics Technology Letters 23 (2011) 1219-1221.

[22] C. Zhong, C. Shen, Y. You, J. Chu, X. Zou, X. Dong, Y. Jin, J. Wang, A polarizationmaintaining fiber loop mirror based sensor for liquid refractive index absolute measurement, Sensors and Actuators B: Chemical 168 (2012) 360-364.

[23] C. Jesus, P. Caldas, O. Frazao, J.L. Santos, P. Jorge, J.M. Baptista, Simultaneous measurement of refractive index and temperature using a hybrid fiber Bragg grating/long-period fiber grating configuration, Fiber and Integrated Optics 28 (6) (2009) 440-449.

[24] S. Silva, J.L. Santos, F.X. Malcata, J. Kobelke, K. Schuster, O. Frazao, Optical refractometer based on large-core air-clad photonic crystal fibers, Optics Letters 36 (6) (2011) 852-854.

[25] M.I. Zibaii, A. Kazemi, H. Latifi, M.K. Azar, S.M. Hosseini, M.H. Ghezelaiagh, Measuring bacterial growth by refractive index tapered fiber optic biosensor, Journal of Photochemistry and Photobiology B: Biology 101 (December (3)) (2010) 313-320.

[26] M.I. Zibaii, H. Latifi, M. Karami, M. Gholami, S.M. Hosseini, M.H. Ghezelayagh, Non-adiabatic tapered optical fiber sensor for measuring the interaction between alpha-amino acids in aqueous carbohydrate solution, Measurement Science and Technology 21 (10) (2010) 105801.

\section{Biographies}

Carlos Gouveia graduated with a degree in electronics and telecommunication engineering, in 2007, and the MSc degree in telecommunications and networks, in 2008 , from the University of Madeira, Portugal. Currently, he is a PhD student in University of Madeira; working in the Optoelectronics Group of INESC Porto. His research interests are on optical fiber sensors and interrogation systems for chemical and biochemical applications.

Giancarlo Chesini graduated with a degree in physics from the University of Campinas (UNICAMP), Campinas, Brazil, in 2006, and the MSc degree in physics, in 2009 from UNICAMP. Currently, he is a PhD student at UNICAMP, working in the Laboratory of Specialty Fibers and Photonics Materials. His research interests are on birefringent optical fibers and electric current/magnetic field sensors.

Cristiano M.B. Cordeiro is assistant professor in the Institute of Physics of the State University of Campinas (UNICAMP), Campinas, Brazil, with PhD in the same University and postdoc from University of Bath, England. Cristiano is head of the Specialty Optical Fiber \& Photonics Materials Laboratory (LaFE) at UNICAMP. The main research areas of the laboratory are the development and application of silica photonic crystal fibers, microstructured polymer optical fibers and micro/nanofibers. Topics of interest also include the waveguide structural and material post-processing for exploring new fiber functionalities. Applications related 
with optical devices and sensors, telecommunications and nanophotonics are under investigation.

J.M. Baptista graduated with a degree in electrical and computer engineering (telecommunications and computers) from the University of Porto, Porto, Portugal, in 1991, the MSc degree in physics of laser communications from the University of Essex, Colchester, UK, in 1993, and the PhD degree in electrical and computer engineering from the University of Porto, in 2002. Currently, he is an associate professor and president of the Exact Sciences and Engineering Competence Center at the University of Madeira and a senior researcher in the Optoelectronics and Electronics Systems Unit at INESC Porto. He is author of 62 journal publications and of two patents. His research interests are in the area of fiber-optic sensors, fiber-optic communications, and fiber-optic technologies.

Pedro A.S. Jorge graduated in applied physics (optics and lasers) from the University of Minho in 1996. He received his MSc in optoelectronics and lasers from the physics department of the University of Porto in 2000. In 2006, he concluded his $\mathrm{PhD}$ program at Porto University in collaboration with the department of physics and optical sciences at the University of Charlotte, NC, USA, with work developed in luminescence based optical fiber. He is currently a senior researcher at INESC Porto, where he leads a team in the development of biochemical sensors for environmental and medical applications. 